\title{
FRITZ CARLSON'S INEQUALITY AND ITS APPLICATION
}

\author{
AMIR KAMALY
}

\begin{abstract}
A Carlson-type inequality is proved and it is applied to show a Babenko-Beckner type of the Hausdorff-Young inequality on $n$-dimensional torus.
\end{abstract}

\section{Introduction}

Fritz Carlson's inequality (1934) states, [4], that

$$
\sum_{n=1}^{\infty} a_{n}<\sqrt{\pi}\left(\sum_{n=1}^{\infty} a_{n}^{2}\right)^{\frac{1}{4}}\left(\sum_{n=1}^{\infty} n^{2} a_{n}^{2}\right)^{\frac{1}{4}}
$$

holds for any positive sequence $\left(a_{n}\right)_{n=1}^{\infty}$ and not all $a_{n}$ are 0 . Let $a_{n}:=\widehat{f}(n)$, for a periodic function $f$. Then, there can be equality only if $f$ is a multiple of $f^{\prime}$, and therefor an exponential function $C_{0} e^{b x}$. This is plainly impossible, [7].

Note that the sums $\sum_{n=1}^{\infty} a_{n}{ }^{2}$ and $\sum_{n=1}^{\infty} n^{2} a_{n}{ }^{2}$ are supposed to be finite.

The corresponding integral inequality, [4], [7], is

$$
\int_{0}^{\infty} f(x) d x \leq \sqrt{\pi}\left(\int_{0}^{\infty} f^{2}(x) d x\right)^{\frac{1}{4}}\left(\int_{0}^{\infty} x^{2} f^{2}(x) d x\right)^{\frac{1}{4}} .
$$

Here there is equality when $f(x):=\frac{1}{a+b x^{2}}$, for any positive $a, b$.

For $f \in A(\mathrm{~T})$ and $\widehat{f}(0)=0$, the other expression of Carlson's inequality is

$$
\|f\|_{A(\mathrm{~T})} \leq C\left(\|f\|_{2}\left\|f^{\prime}\right\|_{2}\right)^{\frac{1}{2}} .
$$

Here $\|f\|_{A(\mathrm{~T})}:=\sum_{m \in Z}|\widehat{f}(m)|$ and $A(\mathrm{~T})$ is the space of continuous functions on $T$ having an absolutely convergent Fourier series. The variety of the constant $C$ in (1) depends on the definitions of T and the Fourier series of $f$. 
B. Kjellberg, [11], and D. Müller, [14] (Lemma 3.1) proved a multidimensional extension of Carlson's inequality of the integral type. By using the idea ${ }^{1}$ of Theorem 2.7.6. in [15], Carlson's inequality can be carried over from $\mathrm{R}^{n}$ to $\mathrm{T}^{n}$. Our proof of the multi-dimensional case of (1) (for the case $\mathrm{R}^{n}$ see [10]) is new and more direct.

The well-known classical Hausdorff-Young inequality (1912-1923) states that, for any complex-valued function $g$ in the Banach space $L^{p}(\mathbf{T})$,

$$
\|\widehat{g}\|_{p^{\prime}} \leq\|g\|_{p}
$$

holds for $1 \leq p \leq 2$. Here and throughout the paper, $p^{\prime}$ is the dual exponent of $p$. Also, $\|\widehat{g}\|_{p^{\prime}}:=\left(\sum_{n \in \mathrm{Z}}|\widehat{g}(n)|^{p^{\prime}}\right)^{\frac{1}{p^{\prime}}}$ and $\|g\|_{p}:=\left(\int_{\mathrm{T}}|g(x)|^{p} d x\right)^{\frac{1}{p}}$ are supposed to be finite.

Titchmarsh, [18], proved (2) for the space $L^{p}(\mathrm{R})$ in 1924. In fact, (2) is true for locally compact unimodular groups , [13]. The result is due to R.A. Kunze (1957). Hardy and Littlewood, [8], showed that (2) is sharp and there is equality if and only if $g=C_{0} e^{2 \pi m i x}$ for $m \in \mathbf{Z}$.

For the space $L^{p}\left(\mathrm{R}^{n}\right)$ and for the even integer $p^{\prime}$, [2], the improvement is due to K.I. Babenko (1961) and for all p, [3], it is due to W. Beckner (1975). That is

$$
\|\widehat{f}\|_{p^{\prime}} \leq B_{p}{ }^{n}\|f\|_{p}
$$

holds for $p \in[1,2] . \quad B_{p}:=\sqrt{\frac{p^{\frac{1}{p}}}{p^{t^{p^{\prime}}}}}$ is called the Babenko-Beckner constant. $\widehat{f}(\xi):=\int_{\mathrm{R}^{n}} f(x) e^{-2 \pi i<\xi, x>} d x$ is the Fourier transform of $f$ and $\langle\xi, x\rangle:=$ $\sum_{\nu=1}^{n} \xi_{\nu} x_{\nu}$.

B. Russo (1974), [16], and J.J.F. Fournier (1977), [6], proved (3) for certain classes of locally compact unimodular groups.

The extension of (3) is due to J. Inoue (1992), [9]. For certain classes of nilpotent Lie groups he improved (3) and obtained the constant

$$
B_{p}{ }^{\operatorname{dim}(G)-\frac{m}{2}} .
$$

Here $G:=\exp (\mathrm{g})$ and $\mathrm{g}$ is Lie algebras with the dual space $\widehat{\mathrm{g}}$. $\operatorname{dim}(G)$ is the dimension of nilpotent Lie groups $G$ and $m$ is the dimension of generic coadjoint orbits of $G$ in $\widehat{\mathrm{g}}$.

For the even integer $p^{\prime}$, [1], M.E. Andersson (1994) and for all $p$, [17], P.

\footnotetext{
${ }^{1}$ The referee made kindly this idea clear to me. He also informed me of the references [11] and [14] and gave me valuable comments on this paper (see the remark).
} 
Sjölin (1995) proved a Babenko-Beckner type inequality (3) for functions in the space $L^{p}(\mathrm{~T})$, with small supports.

The purpose of this paper is to prove Carlson's inequality of type (1) on $n$ dimensional torus and applying it to prove a Babenko-Beckner type of the Hausdorff-Young inequality for periodic functions with small supports.

\section{Theorems and Proofs}

Let the multi-indices $\beta$ and $\gamma$ be vectors in $\mathrm{R}^{n}$ with components $\beta_{k}$ and $\gamma_{k}$ in $\mathrm{N}_{0}$ such that $\gamma \leq \beta$ is equivalent to $\gamma_{k} \leq \beta_{k}$ for all $1 \leq k \leq n$. Define $m^{\beta}:=\prod_{k=1}^{n} m_{k}^{\beta_{k}}$ for $m \in Z^{n}$ and $0^{0}:=1$.

Throughout this paper, $|\beta|:=\sum_{k=1}^{n} \beta_{k}$ and $\beta \gamma:=\prod_{k=1}^{n} \beta_{k} \gamma_{k}$. The operator $D^{\beta}:=\prod_{k=1}^{n} \frac{\partial^{\beta_{k}}}{\partial x_{k} \beta_{k}}$.

Let also

$$
H_{p, a}:=\sup \left\{\frac{\|\widehat{g}\|_{p^{\prime}}}{\|g\|_{p}}: g \in L^{p}\left(\mathrm{~T}^{n}\right), \text { supp } g \subset \bar{B}(0, a),\|g\|_{p} \neq 0\right\}
$$

and define $H_{p}:=\lim _{a \rightarrow 0^{+}} H_{p, a}$. Here and everywhere in the paper $a$ obeys the restriction $0<a<\frac{1}{2}$ and $\bar{B}(0, a)$ is a closed ball of radius $a$, centered at the origin. Also, $\mathrm{T}^{n}:=\left\{x \in \mathrm{R}^{n}:\left|x_{\nu}\right| \leq \frac{1}{2}, 1 \leq \nu \leq n\right\}$.

Assume $\varphi(x):=\left\{\begin{array}{ll}1 & |x| \leq \frac{1}{2} \\ 0 & |x| \geq 1\end{array}\right.$ such that $\varphi \in C_{0}^{\infty}\left(\mathrm{R}^{n}\right), \quad 0 \leq \varphi \leq 1$ and $\varphi_{a}:=\varphi\left(\frac{x}{a}\right)$. Define $\Psi(x)=\left(e^{-2 \pi i<b, x>}-1\right) \varphi_{a}(x)$. Here $b:=\left(b_{1}, b_{2}, \cdots, b_{n}\right)$ and $\left|b_{k}\right| \leq \frac{1}{2}, 1 \leq k \leq n$.

With the previous notation, we prove the following:

THeORem 1 (Generalisation of Carlson's inequality). Let $f \in A\left(\mathrm{~T}^{n}\right)$ and $\widehat{f}(0)=0$. Let the absolute value of the multi-index $\beta$ be equal to the positive integer $\alpha$ such that $\alpha \geq 1$ and $\alpha>\frac{n}{q}$ where $1<q \leq 2$. Then we get

$$
\|f\|_{A\left(\mathrm{~T}^{n}\right)} \leq K_{n, q}^{(\alpha)}\|f\|_{q}{ }^{1-\frac{n}{q \alpha}}\left(\sum_{|\beta|=\alpha}\left\|D^{\beta} f\right\|_{q}\right)^{\frac{n}{q \alpha}}
$$

In the case $\widehat{f}(0) \neq 0$, we obtain

$$
\|f\|_{A\left(\mathrm{~T}^{n}\right)} \leq\|f\|_{1}+K_{n, q}^{(\alpha)}\|f\|_{q}^{1-\frac{n}{q \alpha}}\left(\sum_{|\beta|=\alpha}\left\|D^{\beta} f\right\|_{q}\right)^{\frac{n}{q \alpha}} .
$$

The positive constant $K_{n, q}^{(\alpha)}$ depends only on $n, \alpha$ and $q$.

Proof of Theorem 1. The technique is analogous to the case $n=1$, due to Hardy, [7]. Let $\widehat{f}(0)=0$ and $q^{\prime}$ be the dual exponent of $q$. Define 


$$
\begin{aligned}
S & :=\|\widehat{f}\|_{q^{\prime}}^{q^{\prime}} \\
T & :=\sum_{|\beta|=\alpha}\left\|\widehat{D^{\beta} f}\right\|_{q^{\prime}}^{q^{\prime}} .
\end{aligned}
$$

For $t>0$ we also define

$$
P:=\sum_{|\beta|=\alpha}\left(t+\left|(2 \pi m)^{\beta}\right|^{q^{\prime}}\right)
$$

Then $T \leq\left(\sum_{|\beta|=\alpha}\left\|\widehat{D^{\beta} f}\right\|_{q^{\prime}}\right)^{q^{\prime}}$.

By Hölder's inequality we get

$$
\begin{aligned}
\|f\|_{A\left(T^{n}\right)} & =\sum_{|m|>0} \widehat{f}(m) \mid P^{\frac{1}{q^{\prime}}} P^{-\frac{1}{q^{\prime}}} \\
& \leq\left(\sum_{|m|>0}|\widehat{f}(m)|^{q^{\prime}} P\right)^{\frac{1}{q^{\prime}}}\left(\sum_{|m|>0} P^{-\frac{q}{q^{\prime}}}\right)^{\frac{1}{q}} \\
& \leq t^{-\frac{1}{q^{\prime}}}\left(t c_{n, \alpha} S+T\right)^{\frac{1}{q^{\prime}}}\left[\sum_{|m|>0}\left(1+\frac{C_{n, \alpha}}{t}|m|^{q^{\prime} \alpha}\right)^{-\frac{q}{q^{\prime}}}\right]^{\frac{1}{q}} .
\end{aligned}
$$

Because

$$
\sum_{|\beta|=\alpha}\left(t+\left|(2 \pi m)^{\beta}\right|^{q^{\prime}}\right)=c_{n, \alpha} t+\sum_{|\beta|=\alpha}\left|(2 \pi m)^{\beta}\right|^{q^{\prime}} \geq t+t C_{n, \alpha}|m|^{q^{\prime} \alpha} .
$$

Here $c_{n, \alpha}:=\sum_{|\beta|=\alpha} 1$ and $\widehat{D^{\beta} f}(m)=(2 \pi i m)^{\beta} \widehat{f}(m)$. The positive constant $C_{n, \alpha}$ does depend on $n$ and $\alpha$.

It is not hard to see that the sum $\left[\sum_{|m|>0} \frac{1}{\left(1+|m|^{q^{\prime} \alpha}\right)^{\frac{q}{q^{\prime}}}}\right]^{\frac{1}{q}}$ is finite for $\alpha>\frac{n}{q}$ and

$$
\int_{0}^{\infty} \frac{d x}{\left(1+x^{\frac{q^{\prime} \alpha}{n}}\right)^{\frac{q}{q^{\prime}}}}=\frac{\Gamma\left(\frac{n(q-1)}{q \alpha}\right) \Gamma\left(\frac{(q-1)(q \alpha-n)}{q \alpha}\right)}{\frac{q \alpha}{n(q-1)} \Gamma(q-1)} .
$$

Now, by (4.1) and (4.2) we obtain 


$$
\begin{aligned}
\|f\|_{A\left(\mathrm{~T}^{n}\right)} & \leq c_{0} t^{-\frac{1}{q^{\prime}}}\left(t c_{n, \alpha} S+T\right)^{\frac{1}{q^{\prime}}}\left[\int_{\mathrm{R}^{n}} \frac{d x}{\left(1+\frac{C_{n, \alpha}}{t}|x|^{q^{\prime} \alpha}\right)^{\frac{q}{q^{\prime}}}}\right]^{\frac{1}{q}} \\
& =c_{0} t^{-\frac{1}{q^{\prime}}}\left(\frac{t}{C_{n, \alpha}}\right)^{\frac{n}{q q^{\prime} \alpha}}\left(t c_{n, \alpha} S+T\right)^{\frac{1}{q^{\prime}}}\left(\int_{\mathrm{R}^{n}} \frac{d x}{\left(1+|x|^{q^{\prime} \alpha}\right)^{\frac{q}{q^{\prime}}}}\right)^{\frac{1}{q}} \\
& =c_{0} t^{-\frac{1}{q^{\prime}}}\left(\frac{t}{C_{n, \alpha}}\right)^{\frac{n}{q q^{\prime} \alpha}}\left(t c_{n, \alpha} S+T\right)^{\frac{1}{q^{\prime}}}\left(\int_{0}^{\infty} \int_{\left\{x \in \mathrm{R}^{n-1}:|x|=1\right\}} \frac{r^{n-1} d r d x}{\left(1+r^{q^{\prime} \alpha}\right)^{\frac{q}{q^{\prime}}}}\right)^{\frac{1}{q}} \\
& =c_{0}\left(\frac{w_{n-1}}{n}\right)^{\frac{1}{q}} t^{-\frac{1}{q^{\prime}}}\left(\frac{t}{C_{n, \alpha}}\right)^{\frac{1}{q q^{\prime} \alpha}}\left(t c_{n, \alpha} S+T\right)^{\frac{1}{q^{\prime}}}\left(\int_{0}^{\infty} \frac{d x}{\left(1+x^{\frac{q^{\prime} \alpha}{n}}\right)^{\frac{q}{q^{\prime}}}}\right)^{\frac{1}{(\alpha)}} \\
& =c_{0} A_{n, q}^{(\alpha)} \frac{n q^{\prime}}{t^{\frac{1}{q}}}\left(c_{n, \alpha} S+\frac{T}{t}\right)^{\frac{1}{q^{\prime}}},
\end{aligned}
$$

for a positive constant $c_{0}$. Here

$$
A_{n, q}^{(\alpha)}:=\sqrt[q]{\frac{(q-1) w_{n-1} \Gamma\left(\frac{n(q-1)}{q \alpha}\right) \Gamma\left(\frac{(q-1)(q \alpha-n)}{q \alpha}\right)}{q \alpha \Gamma(q-1)}\left(C_{n, \alpha}\right)^{\frac{n(1-q)}{q \alpha}}},
$$

and $w_{n-1}$ is the surface area of the unit sphere in $\mathrm{R}^{n-1}$.

Choose $t=\frac{S}{T}$, then by using (two times) the classical Hausdorff-Young inequality (2) we get

$$
\begin{aligned}
\|f\|_{A\left(\mathrm{~T}^{n}\right)} & \leq c_{0} A_{n, q}^{(\alpha)} \sqrt[q]{\left(c_{n, \alpha}+1\right)^{q-1}}\|\widehat{f}\|_{q^{\prime}}{ }^{1-\frac{n}{q \alpha}}\left(\sum_{|\beta|=\alpha}\left\|\widehat{D^{\beta} f}\right\|_{q^{\prime}}\right)^{\frac{n}{q \alpha}} \\
& \leq c_{0} A_{n, q}^{(\alpha)} \sqrt[q]{\left(c_{n, \alpha}+1\right)^{q-1}}\|f\|_{q}^{1-\frac{n}{q \alpha}}\left(\sum_{|\beta|=\alpha}\left\|D^{\beta} f\right\|_{q}\right)^{\frac{n}{q \alpha}} \\
& =K_{n, q}^{(\alpha)}\|f\|_{q}^{1-\frac{n}{q \alpha}}\left(\sum_{|\beta|=\alpha}\left\|D^{\beta} f\right\|_{q}\right)^{\frac{n}{q \alpha}}
\end{aligned}
$$

For the case $\widehat{f}(0) \neq 0$ the proof is similar and we know that $|\widehat{f}(0)| \leq\|f\|_{1}$. 
Application of Theorem 1 for estimating of the $A\left(\mathrm{~T}^{n}\right)$-norm of $\Psi$ and $H_{p, a}$

Lemma (An upper bound for $\|\Psi\|_{A\left(\mathrm{~T}^{n}\right)}$ ). There exists a positive constant $C_{0}$, does not depend on a, such that

$$
\|\Psi\|_{A\left(\mathrm{~T}^{n}\right)} \leq C_{\mathrm{o}} a .
$$

Proof of Lemma. It is obvious that $\Psi \in C_{0}{ }^{\infty}\left(\mathrm{R}^{n}\right)$ and for $m \in \mathrm{Z}^{n}$ we get

$$
\begin{aligned}
|\widehat{\Psi}(m)| & =\left|\int_{|x| \leq a} \Psi(x) e^{-2 \pi i<m, x>} d x\right| \leq a^{n} \int_{|y| \leq 1}\left|e^{-2 \pi i a<b, y>}-1\right| d y \\
& \leq \pi \sqrt{n} a^{n+1} \int_{|y| \leq 1} d x={ }_{n} a^{n+1}
\end{aligned}
$$

because

$$
\left|e^{-2 \pi i a<b, y>}-1\right| \leq 2 \pi a \mid<b, y>\leq \pi \sqrt{n} a .
$$
$\mathrm{R}^{n}$.

Here $\Omega_{n}=\sqrt{n} \pi w_{n}$ and $w_{n}=\frac{2 \pi^{\frac{n}{2}}}{\Gamma\left(\frac{n}{2}\right)}$ is the surface area of the unit sphere in

Furthermore, by Leibniz's formula, together with Minkowski's inequality we obtain

$$
\begin{aligned}
& \sum_{|\beta|=\alpha}\left\|D^{\beta} \Psi\right\|_{q} \leq \pi \sqrt{n} a \sum_{|\beta|=\alpha}\left\|D^{\beta} \varphi_{a}\right\|_{q}+\sum_{|\beta|=\alpha} \sum_{\substack{\gamma \leq \beta \\
|\gamma| \neq}}\left(\begin{array}{c}
\beta \\
\gamma
\end{array}\right) \pi^{|\gamma|}\left\|D^{\beta-\gamma} \varphi_{a}\right\|_{q} \\
& \leq \pi \sqrt{n} a^{1-\alpha+\frac{n}{q}} \sum_{|\beta|=\alpha}\left\|D^{\beta} \varphi\right\|_{q}+\sum_{|\beta|=\alpha} \sum_{\substack{\gamma \leq \beta \\
\gamma \mid \neq 0}}\left(\begin{array}{c}
\beta \\
\gamma
\end{array}\right) \pi^{|\gamma|} a^{|\gamma|-|\beta|+\frac{n}{q}}\left\|D^{\beta-\gamma} \varphi\right\|_{q} \\
& \leq a^{1-\alpha+\frac{n}{q}}\left\{\pi \sqrt{n} \sum_{|\beta|=\alpha}\left\|D^{\beta} \varphi\right\|_{q}+\sum_{|\beta|=\alpha} \sum_{\substack{\gamma \leq \beta \\
|\gamma| \neq 0}}\left(\begin{array}{c}
\beta \\
\gamma
\end{array}\right) \pi^{|\gamma|}\left\|D^{\beta-\gamma} \varphi\right\|_{q}\right\} \\
& =A_{n, q, \alpha} a^{1-\alpha+\frac{n}{q}}, 5
\end{aligned}
$$

because

$$
\sum_{|\beta|=\alpha}\left\|D^{\beta} \varphi_{a}\right\|_{q}=a^{\frac{n}{q}-\alpha} \sum_{|\beta|=\alpha}\left\|D^{\beta} \varphi\right\|_{q}
$$

Now, by Theorem 1 and invoking (5), we get 


$$
\begin{aligned}
\|\widehat{\Psi}\|_{1} & =\sum_{m \in Z^{n}}|\widehat{\Psi}(m)| \leq \Omega_{n} a^{n+1}+\sum_{|m|>0}|\widehat{\Psi}(m)| \\
& \leq \Omega_{n} a^{n+1}+K_{n, q}^{(\alpha)}\|\Psi\|_{q}{ }^{1-\frac{n}{q \alpha}}\left(\sum_{|\beta|=\alpha}\left\|D^{\beta} \Psi\right\|_{q}\right)^{\frac{n}{q \alpha}} \\
& \leq \Omega_{n} a^{n+1}+\left[K_{n, q}^{(\alpha)}\left(\pi \sqrt{n}\|\varphi\|_{q}\right)^{1-\frac{n}{q \alpha}} A_{n, q, \alpha}^{\frac{n}{q \alpha}}\right] a \\
& \leq\left\{\Omega_{n}+K_{n, q}^{(\alpha)} A_{n, q, \alpha}{ }^{\frac{n}{q \alpha}}\left(\pi \sqrt{n}\left\|_{\varphi}\right\|_{q}\right)^{1-\frac{n}{q \alpha}}\right\} a \\
& =C_{0} a,
\end{aligned}
$$

because

$$
\|\Psi\|_{q} \leq \pi \sqrt{n}\|\varphi\|_{q} a^{1+\frac{n}{p}}
$$

Note that $\alpha$ is the positive integer defined in Theorem 1 and $\|\widehat{\Psi}\|_{1}:=\|\Psi\|_{A\left(\mathrm{~T}^{n}\right)}$.

Theorem 2 (An upper bound for $H_{p, a}$ ). For a fixed $n \in \mathrm{N}$, there exists a positive constant $C_{0}$ which does not depend on a, such that

$$
H_{p, a} \leq\left(1+C_{0} a\right) B_{p}{ }^{n}, 1 \leq \mathrm{p} \leq 2 .
$$

Proof of Theorem 2. The technique is analogous to the case $n=1$, due to Y. Domar, [5]. Choose $f \in L^{p}\left(\mathrm{R}^{n}\right), \quad g \in L^{p}\left(\mathrm{~T}^{n}\right)$, such that $f=g$ on the ball $\bar{B}(0, a)$ and zero outside of the ball. Define $g_{b}(x):=e^{-2 \pi i<x, b>} g(x)$. Then

$$
\begin{aligned}
\widehat{g_{b}}(m) & =\widehat{g}(m+b) \\
\|f\|_{p} & =\|g\|_{p} \\
\widehat{f_{b}}(m) & =\widehat{g_{b}}(m) .
\end{aligned}
$$

Also, we get

$$
\begin{aligned}
\widehat{g_{b}}(m)-\widehat{g}(m) & =\int e^{-2 \pi i\langle m, x\rangle}\left(e^{-2 \pi i\langle b, x\rangle}-1\right) g(x) d x \\
& \leq \int_{\bar{B}(0, a)} \Psi(x) g(x) e^{-2 \pi i\langle m, x\rangle} d x \\
& =\int_{\bar{B}(0, a)} g(x)\left(\sum_{m^{\prime} \in \mathbf{Z}^{n}} \widehat{\Psi}\left(m^{\prime}\right) e^{2 \pi i\left\langle m^{\prime}, x\right\rangle}\right) e^{-2 \pi i\langle m, x\rangle} d x \\
& =\sum_{m^{\prime} \in \mathbf{Z}^{n}} \widehat{\Psi}\left(m^{\prime}\right) \widehat{g}\left(m-m^{\prime}\right) .
\end{aligned}
$$


Thus, we obtain

$$
\left\|\widehat{g_{b}}-\widehat{g}\right\|_{p^{\prime}} \leq\left(\sum_{m^{\prime} \in Z^{n}}\left|\widehat{\Psi}\left(m^{\prime}\right)\right|\right)\left(\sum_{m \in Z^{n}}|\widehat{g}(m)|^{p^{\prime}}\right)^{\frac{1}{p^{\prime}}}=\|\widehat{g}\|\left\|_{p^{\prime}}\right\| \widehat{\Psi} \|_{1} .
$$

By triangle inequality we have

$$
\|\widehat{g}\|_{p^{\prime}}-\left\|\widehat{g_{b}}\right\|_{p^{\prime}} \leq\left\|\widehat{g_{b}}-\widehat{g}\right\|_{p^{\prime}} \leq\|\widehat{g}\|_{p^{\prime}}\|\widehat{\Psi}\|_{1} .
$$

Similarly, for $t \in \mathrm{R}^{n}$, we obtain

$$
\|\widehat{g}\|_{p^{\prime}}\left(1-\|\widehat{\Psi}\|_{1}\right) \leq\left\|\widehat{g_{b}}\right\|_{p^{\prime}}=\left(\sum_{m \in Z^{n}}\left|\widehat{g_{b}}(m)\right|^{p^{\prime}}\right)^{\frac{1}{p^{\prime}}}=\left(\sum_{m}\left|\widehat{f_{b}}(m)\right|^{p^{\prime}}\right)^{\frac{1}{p^{\prime}}} .
$$

That is

$$
\begin{aligned}
\|\widehat{g}\|_{p^{\prime}}\left(1-\|\widehat{\Psi}\|_{1}\right) & \leq\left(\sum_{m} \int_{\left\{b:\left|b_{k}\right| \leq \frac{1}{2}\right\}}\left|\widehat{f}_{b}(m)\right|^{p^{\prime}} d b\right)^{\frac{1}{p^{\prime}}} \\
& =\left(\sum_{m} \int_{\left\{t-m:\left|t_{k}-m_{k}\right| \leq \frac{1}{2}\right\}}|\widehat{f}(t)|^{p^{\prime}} d t\right)^{\frac{1}{p^{\prime}}} \\
& =\|\widehat{f}\|_{p^{\prime}} .
\end{aligned}
$$

Now, by Lemma we get

$$
\|\widehat{g}\|_{p^{\prime}} \leq \frac{\|\widehat{f}\|_{p^{\prime}}}{1-\|\widehat{\Psi}\|_{1}} \leq \frac{\|\widehat{f}\|_{p^{\prime}}}{1-C_{0} a}
$$

By (6), thus, we obtain

$$
H_{p, a}=\sup _{g} \frac{\|\widehat{g}\|_{p^{\prime}}}{\|g\|_{p}} \leq \frac{B_{p}{ }^{n}}{1-C_{0} a} .
$$

Because

$$
\sup _{f} \frac{\|\widehat{f}\|_{p^{\prime}}}{\|f\|_{p}}=B_{p}{ }^{n}
$$

(see [3], p. 160). Choose $a$ such that $C_{0} a<\frac{1}{2}$, then we get

$$
\frac{1}{1-C_{0} a} \approx 1+C_{0} a
$$

because $\frac{1}{1-C_{0} a}=1+C_{0} a+O\left(C_{0}^{2} a^{2}\right)$. Hence

$$
H_{p, a} \leq\left(1+C_{0} a\right) B_{p}{ }^{n}
$$


REMARK. The arguments in this proof can be used to prove that the quotient of the norms $\widehat{g}$ and $\widehat{f}$ in $l^{p^{\prime}}$ and $L^{p^{\prime}}$, respectively, is $1+O(a)$, as $a \rightarrow 0^{+}$.

\section{The Babenko-Beckner type of the Hausdorff-Young inequality for periodic functions with small supports}

THEOREM $3 H_{p} \leq B_{p}{ }^{n}$.

The proof is a consequence of Theorem 2 .

\section{REFERENCES}

1. M.E. Andersson, Local variants of the Hausdorff-Young inequality, Gyllenberg, Persson (Eds.) Analysis, Algebra and Computers in Mathematical Research: Proceedings of the 21 st Nordic congress of mathematicians (1994), Marcel-Dekker, New York.

2. K.I. Babenko, An inequality in theory of Fourier integrals, Amer. Math. Soc. Transl. (2) 44 (1965), 115-128.

3. W. Beckner, Inequalities in Fourier integrals, Ann. of Math. 102 (1975), 159-182.

4. F. Carlson, Une inégalité, Arkiv för matematik, astronomi och fysik 25 B, No. 1 (1934).

5. Y. Domar, private communication with P. Sjölin.

6. J.J. Fournier, Sharpness in Young's inequality for convolution, Pacific J. Math. 72, No. 2 (1974), 293-397.

7. G.H. Hardy, A note on two inequalities, J. London Math.Soc. 11 (1936), 167-170.

8. G.H. Hardy, J.E. Littlewood, G. Pólya, Inequalities, Cambridge University Press, London J. London (1934).

9. J. Inoue, $L^{p}$-Fourier transforms on nilpotent Lie groups and solvable Lie groups action on Siegel domains, Pacific J. Math. 155, No. 2 (1992), 295-318.

10. A. Kamaly, Some new inequalities for integral and discrete norms, Unpubished (1996).

11. B. Kjellberg, Ein Momentenproblem, Arkiv för matematik, astronomi och fysik 29 A, No. 2 (1942).

12. N.Y. Krugljak, L. Maligranda and L.E. Persson, A Carlson type inequality with blocks and interpolation, Studia Math. 104, No. 2 (1993), 161-180.

13. R.A. Kunze, $L^{p}$-Fourier transforms on locally copmact unimodular groups, Trans. Amer. Math. Soc. 89 (1958), 519-540.

14. D. Müller, On the Spectral Synthesis Problem for Hypersurfaces of $\mathbf{R}^{n}$, J. Funct. Anal. 47 (1982), 247-280.

15. W. Rudin, Fourier Analysis on Groups, Interscience Publishers, London (1962).

16. B. Russo, The norm of the $L^{p}$-Fourier transform on unimodular groups, Trans. Amer. Math. Soc. 192 (1974), 293-318.

17. P. Sjölin, A remark on the Hausdorff-Young inequality, Proc. Amer. Math. Soc. 123 (Oct. 1995), 3085-3088.

18. E.C. Titchmarsh, Introduction to the Theory of Fourier Integrals (1937), Oxford, at Clarendon Press. 